\title{
Caution is essential when using a tracheal tube as a stabilizing rod to remove the Intubating Laryngeal Airway (ILA ${ }^{\text {TM }}$ ) after tracheal intubation in children
}

\author{
Narasimhan Jagannathan, MD • Melanie F. Kho, MS • \\ David T. Wong, MD
}

Received: 31 March 2010/Accepted: 15 April 2010/Published online: 30 April 2010

(C) Canadian Anesthesiologists' Society 2010

\section{To the Editor,}

Use of a supraglottic airway device as a conduit for tracheal intubation has changed the practice of pediatric anesthesia, especially in the management of patients with difficult airways. For many years, the Laryngeal Mask Airway (LMA) Classic ${ }^{\mathrm{TM}}$ (LMA North America, San Diego, CA, USA) was the device most commonly used for this purpose in pediatric anesthesia. However, there are some limitations when the LMA is used to facilitate fibreoptic intubation with a cuffed endotracheal tube (ETT), particularly during subsequent removal of the LMA. ${ }^{1}$ The Intubating Laryngeal Airway (ILA ${ }^{\mathrm{TM}}$ ) (Cookgas LLC, St. Louis, MO, USA) is a new supraglottic airway device that is comparable to the LMA in terms of its ease of insertion and functionality, but with a conduit sufficient in size to accommodate a larger cuffed ETT. ${ }^{1,2}$ There are no intubating LMAs currently available for patients weighing $<30 \mathrm{~kg}$, and the ILA, which is available in pediatric sizes, can be used to facilitate tracheal intubation in these patients.

A Stabilizer $\operatorname{Rod}^{\mathrm{A}}$ is used to facilitate removal of the LMA after successful tracheal intubation. Like the LMA, the ILA has a Removal Stylet ${ }^{B}$ available to steady the ETT during removal of the ILA device. The ILA lumen is of sufficient size to accommodate the Stylet and the pilot balloon of the ETT during withdrawal of the ILA (Figure 1A-C). In clinical practice, however, an uncuffed ETT is often used in lieu of the manufacturer's removal

N. Jagannathan, MD $(\varangle) \cdot$ M. F. Kho, MS

Children's Memorial Hospital, Northwestern University's

Feinberg School of Medicine, Chicago, IL, USA

e-mail: simjag2000@yahoo.com

D. T. Wong, MD

Toronto Western Hospital, University of Toronto,

Toronto, ON, Canada stylet when removing the LMA or ILA after using either as a conduit for tracheal intubation in children (Figure 1D-F). The ILA lumen cannot accommodate both the pilot balloon and the ETT during ILA withdrawal (Figure 1F), and entrapment of the pilot balloon within the airway tube may lead to cuff line rupture if the ILA is withdrawn further in that situation. The disadvantages of using two tracheal tubes include the potential for cuff line rupture or inadvertent ETT dislodgement upon removal of the ILA. To avoid either situation, control of the distal cuffed ETT must be maintained while the proximal ETT is first removed from the ILA (Figure 1E). After that, the cuff line and the pilot balloon can pass through the ILA airway tube unhindered. This is of particular importance in the management of the difficult airway, where the initial securing of the airway may have been difficult and where re-intubation may be accompanied by untoward problems.

It is convenient to use a second ETT as a stabilizing rod for removal of the ILA after successful tracheal intubation, because an ETT is readily available in the operating room and is used commonly when removing the LMA-Classic after tracheal intubation in pediatric patients. ${ }^{3}$ As the ILA and tracheal intubation with cuffed ETTs ${ }^{4}$ become more common in pediatric anesthesia, awareness of the limitations of using an ETT as a stabilizing rod to assist in removal of the ILA is clinically important. In our clinical experience of more than 100 ILA-guided tracheal intubations, we have used a tracheal tube primarily as a stabilizing rod when removing the ILA. However, we have encountered difficulty when removing the ILA with the use

\footnotetext{
A LMA Fastrach ${ }^{\mathrm{TM}}$ Stabilizer Rod. Available from URL: http:// www.lmana.com/fastrach_rod.php (accessed April 2010).

B Cookgas Removal Stylet. Available from URL: http://www. cookgas.com/stylet.htm (accessed April 2010).
} 

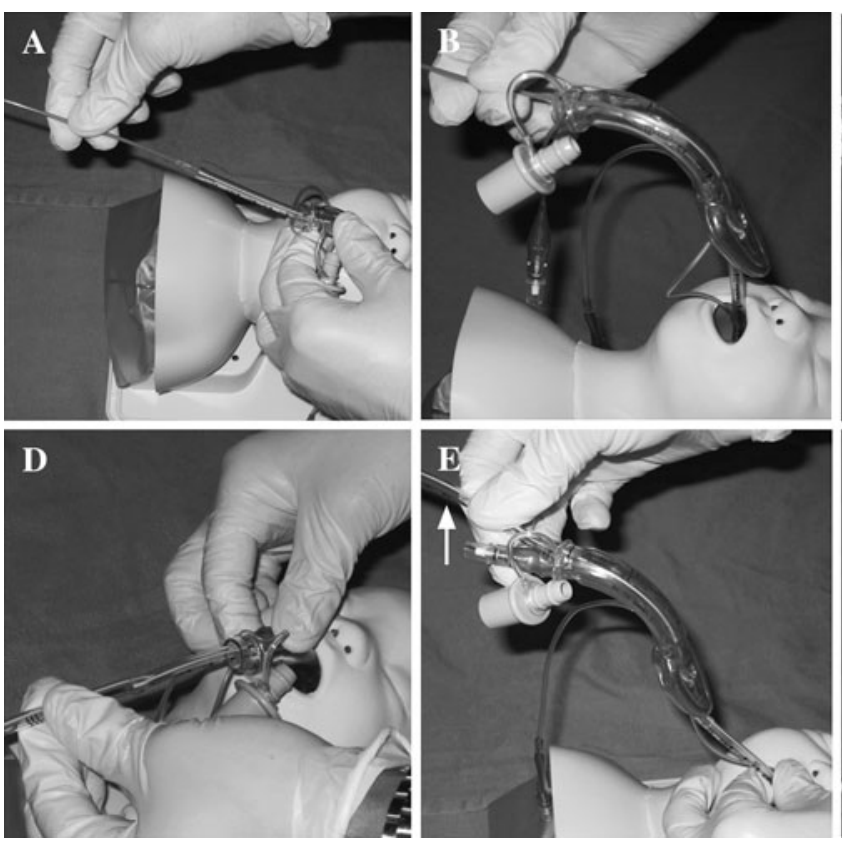
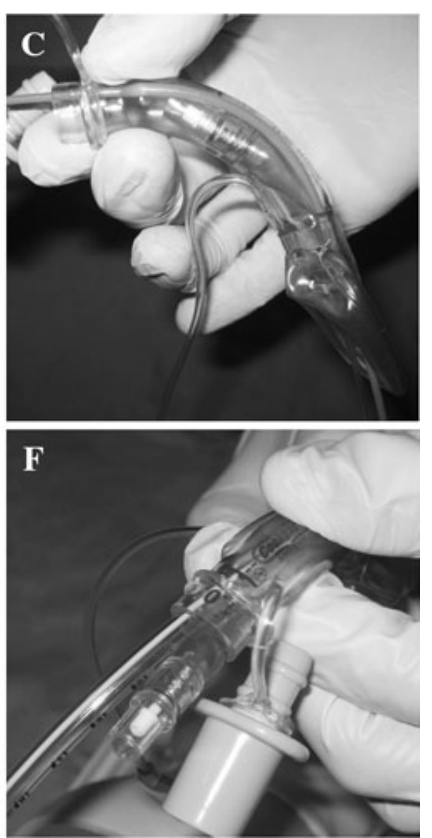

Fig. 1 A-C Use of the Removal Stylet as a stabilizing rod with the Intubating Laryngeal Airway (ILA). (A) Placement of the Removal Stylet in a cuffed tracheal tube after successful tracheal intubation through the ILA. (B) Removal of the ILA using the Removal Stylet to stabilize the cuffed tracheal tube. (C) The airway tube of the ILA allows passage of both the pilot balloon and the Removal Stylet without entrapment. D-F Use of a second uncuffed tracheal tube as a stabilizing rod with the ILA. (D) Connection of the proximal tracheal

of a second ETT. In two cases, it led to pilot balloon rupture necessitating use of an airway exchange catheter to replace the cuffed ETT. These incidents have made us more cognizant when using a second tracheal tube. As a result, we now prefer to use the Removal Stylet in our practice to stabilize the ETT when removing the ILA.

In summary, caution should be exercised when using a tracheal tube as a stabilizing rod to safely remove the ILA after it is used to facilitate tracheal intubation. Use of the Removal Stylet may decrease the potential for complications associated with removing the ILA because it is specifically designed for this purpose.

Disclosure The ILA/air- $\mathrm{Q}^{\mathrm{TM}}$ devices used were generously provided by the manufacturer (Cookgas LLC; St. Louis, MO, USA).

Competing interests None declared. tube to the distal cuffed tracheal tube after successful tracheal intubation through the ILA. (E) Removal of the ILA with distal control over the cuffed tracheal tube. Removal of the proximal tracheal tube (arrow) before further withdrawal of the ILA is recommended to prevent entrapment of the pilot balloon. (F) Entrapment of the pilot balloon and the proximal tracheal tube within the airway tube of the ILA. Further withdrawal of the ILA in this scenario may lead to cuff line rupture

\section{References}

1. Jagannathan N, Roth AG, Sohn LE, Pak TY, Amin S, Suresh S. The new air-Q intubating laryngeal airway for tracheal intubation in children with anticipated difficult airway: a case series. Paediatr Anaesth 2009; 19: 618-22.

2. Wong DT, Zilberman P. Awake intubation through an intubating laryngeal airway $\left(\mathrm{ILA}^{\mathrm{TM}}\right.$ ) in a patient with Still's disease. Can J Anesth 2010; 57: 286-7.

3. Koga K, Asai T, Latto IP, Vaughan RS. Effect of the size of a tracheal tube and the efficacy of the use of the laryngeal mask for fibrescope-aided tracheal intubation. Anaesthesia 1997; 52: 131-5.

4. Weiss M, Dullenkopf A, Fischer JE, Keller C. Gerber AC; European Paediatric Endotracheal Intubating Study Group. Prospective randomized controlled multi-centre trial of cuffed or uncuffed endotracheal tubes in small children. Br J Anaesth 2009; 103: 867-73. 